\title{
Alternatives to Invasive Plants Commonly Found in Central Florida Landscapes ${ }^{1}$
}

\author{
Gary W. Knox, Sandra B. Wilson, Zhanao Deng, and Rosanna Freyre²
}

Invasive plants are non-native plants that form expanding populations in natural areas and other plant communities with which they were not previously associated (Langeland 2012). Invasive plants can cause ecological impacts, such as displacing native plants and associated wildlife or altering natural water flow and fire patterns.

Some ornamentals listed as invasive by the University of Florida IFAS Assessment of Non-Native Plants in Florida's Natural Areas or by the Florida Exotic Pest Plant Council are still in commercial production and widely found in Florida landscapes. Homeowners might replace invasive plants if non-invasive alternatives are researched, publicized, and made readily available. By shifting production and use from invasive ornamentals to native or non-invasive cultivars, the nursery and landscape industry could benefit from potential revenue while fostering greater collaboration with state agencies and environmental groups.

University of Florida research and Extension efforts over the last 10 years have focused on identifying non-invasive alternatives by assessing the invasive traits of popular non-native ornamentals, related genera, and their cultivars. In more recent years, University of Florida breeding efforts have focused on producing and trialing new non-invasive cultivars. Table 1 lists native and non-invasive, non-native ornamentals as alternatives to invasive plants commonly used in Florida landscapes. Only plants considered to be generally available in the nursery trade are listed.
Alternative plants are similar to respective invasive plants as much as possible in terms of size, habit, texture, and flower color. Non-native, non-invasive plants in Table 1 were determined to be non-invasive by the IFAS Assessment of Non-Native Plants in Florida's Natural Areas (IFAS Invasive Plant Working Group 2008) or have not yet been evaluated.

\section{References}

Fox, A. M., D. R. Gordon, J. A. Dusky, L. Tyson, and R. K. Stocker. 2009. IFAS Assessment of Non-Native Plants in Florida's Natural Areas: Status Assessment. Gainesville: University of Florida Institute of Food and Agricultural Sciences. http://plants.ifas.ufl.edu/assessment/pdfs/Final_PDF_SS-AGR-225_04.30.09.pdf.

Fox, A. M., D. R. Gordon, C. Gantz, G. W. Knox, and S. B. Wilson. 2007. IFAS Assessment: Infraspecific Taxon Protocol. Gainesville: University of Florida Institute of Food and Agricultural Sciences. http://plants.ifas.ufl.edu/assessment/ infraspecific_taxon_protocol.html.

IFAS Invasive Plant Working Group. 2008. IFAS Assessment of Non-Native Plants in Florida's Natural Areas. Gainesville: University of Florida Institute of Food and Agricultural Sciences. http://plants.ifas.ufl.edu/assessment/.

Langeland, K. A. 2012. Help Protect Florida's Natural Areas from Non-Native Invasive Plants. Circular 1204. Gainesville: University of Florida Institute of Food and Agricultural Sciences. http://edis.ifas.ufl.edu/ag108

1. This document is ENH1207, one of a series of the Environmental Horticulture Department, UF/IFAS Extension. Original publication date August 2013. Visit the EDIS website at http://edis.ifas.ufl.edu.

2. Gary W. Knox, Extension specialist and professor, Environmental Horticulture Department, North Florida Research and Education Center, Quincy, FL; Sandra B. Wilson, professor, Environmental Horticulture Department, Indian River Research and Education Center, Fort Pierce, FL; Zhanao Deng, associate professor, Environmental Horticulture Department, Gulf Coast Research and Education Center, Wimauma, FL; and Rosanna Freyre, research scientist, Environmental Horticulture Department, University of Florida Institute of Food and Agricultural Sciences, Gainesville, FL. 
Table 1. Invasive ornamentals commonly found in central Florida landscapes and commonly available native and non-native, non-invasive substitutes

\begin{tabular}{|c|c|c|c|}
\hline \multicolumn{2}{|c|}{ Invasive ornamental ${ }^{\mathrm{z}}$} & \multirow[t]{2}{*}{ Native substitute } & \multirow[t]{2}{*}{ Non-native, non-invasive substitute ${ }^{z}$} \\
\hline Scientific name & Common name & & \\
\hline Albizia julibrissin & Mimosa & $\begin{array}{l}\text { Acacia farnesiana, Sweet acacia } \\
\text { Ardisia escallonioides, Marlberry (cold } \\
\text { tender) } \\
\text { Cercis canadensis, Eastern redbud } \\
\text { Chionanthus virginicus, Fringe tree } \\
\text { Hamelia patens, Firebush } \\
\text { Prunus umbellata, Chickasaw plum }\end{array}$ & $\begin{array}{l}\text { Aloysia virgata, Sweet almondshrub } \\
\text { Callistemon citrinus, Red bottlebrush } \\
\text { Calliandra haematocephala, Powderpuff } \\
\text { Handroanthus heptaphyllus (formerly Tabebuia } \\
\text { heptaphylla), Pink trumpet tree } \\
\text { Jatropha integerrima, Peregrina (cold tender) } \\
\text { Lagerstroemia spp., Crapemyrtle } \\
\text { Tabebuia impetiginosa, Purple tabebuia }\end{array}$ \\
\hline Ardisia crenata & Coral ardisia & $\begin{array}{l}\text { Ilex glabra, Gallberry } \\
\text { Ilex vomitoria (dwarf cultivars), Dwarf } \\
\text { yaupon holly } \\
\text { Psychotria nervosa, Wild coffee }\end{array}$ & $\begin{array}{l}\text { Ilex cornuta, Chinese holly } \\
\text { Osmanthus heterophyllus, False holly }\end{array}$ \\
\hline $\begin{array}{l}\text { Casuarina } \\
\text { equisetifolia }\end{array}$ & Australian pine & $\begin{array}{l}\text { Juniperus silicicola, Southern red cedar } \\
\text { Juniperus virginiana, Red cedar } \\
\text { Pinus spp. (native species), Pine } \\
\text { Quercus geminata, Sand live oak } \\
\text { Taxodium distichum var. distichum, } \\
\text { Baldcypress } \\
\text { Taxodium distichum var. imbricarium, } \\
\text { Pondcypress }\end{array}$ & \\
\hline $\begin{array}{l}\text { Cinnamomum } \\
\text { camphora }\end{array}$ & Camphor tree & $\begin{array}{l}\text { Ilex cassine, Dahoon holly } \\
\text { Magnolia grandiflora, Southern magnolia } \\
\text { Magnolia virginiana, Sweet bay } \\
\text { Persea borbonia, Red bay } \\
\text { Quercus virginiana, Live oak } \\
\text { Ulmus alata, Winged elm }\end{array}$ & Ulmus parvifolia, Lacebark elm \\
\hline $\begin{array}{l}\text { Colocasia } \\
\text { esculenta }\end{array}$ & Elephant ear & $\begin{array}{l}\text { Canna flaccida, Golden canna } \\
\text { Pontederia cordata, Pickerelweed } \\
\text { Sagittaria spp. (native species), Arrowhead }\end{array}$ & $\begin{array}{l}\text { Alocasia spp., Elephant ear } \\
\text { Alpinia spp., Shell ginger } \\
\text { Begonia nelumbiifolia, Lotus-leaf begonia } \\
\text { Caladium } \times \text { hortulanum, Caladium } \\
\text { Canna spp., Canna } \\
\text { Hedychium spp., Butterfly ginger } \\
\text { Heliconia spp., Heliconia } \\
\text { Philodendron bipinnatifidum, Selloum philodendron } \\
\text { Strelitzia reginae, Bird-of-paradise } \\
\text { Zingiber zerumbet, Pinecone ginger }\end{array}$ \\
\hline $\begin{array}{l}\text { Dioscorea } \\
\text { bulbifera }\end{array}$ & Air-potato & $\begin{array}{l}\text { Ipomoea alba, Moonflower } \\
\text { Passiflora spp. (native species), Passionvine }\end{array}$ & $\begin{array}{l}\text { Aristolochia maxima, Florida Dutchman's pipe } \\
\text { (See Flowering Vines for Florida (http://edis.ifas.ufl.edu/ } \\
\text { mg097) for additional vines) }\end{array}$ \\
\hline Lantana camara & Lantana & $\begin{array}{l}\text { Evolvulus glomeratus subsp. grandiflorus, } \\
\text { Blue daze } \\
\text { Helianthus debilis, Beach sunflower } \\
\text { Lantana depressa, Pineland lantana (short } \\
\text { lived) } \\
\text { Lantana involucrata, Wild sage } \\
\text { Salvia coccinea, Tropical sage } \\
\text { Stachytarpheta jamaicensis, Porterweed }\end{array}$ & $\begin{array}{l}\text { Euryops (formerly Gamolepis) chrysanthemoides, African } \\
\text { bush daisy } \\
\text { Lantana camara } \mathrm{T} 2 \mathrm{y}, \mathrm{T} 2 \text { lantana (sterile) } \\
\text { Lantana camara } \mathrm{T} 3^{y}, \mathrm{~T} 3 \text { lantana (sterile) } \\
\text { Lantana camara } \mathrm{T} 4^{y}, \mathrm{~T} 4 \text { lantana (sterile) } \\
\text { Lantana camara T9y, T9 lantana (sterile) } \\
\text { Lantana montevidensis, Trailing lantana } \\
\text { Rosa spp., Rose } \\
\text { Salvia greggii, Autumn sage } \\
\text { Salvia splendens, Scarlet sage }\end{array}$ \\
\hline
\end{tabular}




\begin{tabular}{|c|c|c|c|}
\hline \multicolumn{2}{|c|}{ Invasive ornamental $\left.\right|^{z}$} & \multirow[t]{2}{*}{ Native substitute } & \multirow[t]{2}{*}{ Non-native, non-invasive substitute ${ }^{z}$} \\
\hline Scientific name & Common name & & \\
\hline Ligustrum sinense & Chinese privet & $\begin{array}{l}\text { Agarista populifolia, Florida leucothoe } \\
\text { Citharexylum spinosum, Fiddlewood } \\
\text { Hamelia patens, Firebush } \\
\text { Ilex glabra, Gallberry } \\
\text { Illicium floridanum, Florida anise } \\
\text { Illicium parviflorum, Star anise } \\
\text { Itea virginica, Virginia sweetspire } \\
\text { Viburnum obovatum, Walter's viburnum }\end{array}$ & $\begin{array}{l}\text { Acca sellowiana, Feijoa or pineapple guava } \\
\text { Aloysia virgata, Sweet almondshrub } \\
\text { Camellia spp., Camellia } \\
\text { Gardenia jasminoides, Gardenia } \\
\text { Ilex × 'Nellie R. Stevens', Nellie R. Stevens holly } \\
\text { Ilex cornuta, Chinese holly } \\
\text { Leucophyllum frutescens, Texas sage } \\
\text { Viburnum odoratissimum, Sweet viburnum } \\
\text { Viburnum odoratissimum var. awabuki, Awabuki } \\
\text { viburnum } \\
\text { Viburnum suspensum, Sandankwa viburnum }\end{array}$ \\
\hline Lonicera japonica & $\begin{array}{l}\text { Japanese } \\
\text { honeysuckle }\end{array}$ & $\begin{array}{l}\text { Gelsemium sempervirens, Carolina jessamine } \\
\text { Lonicera sempervirens, Coral honeysuckle }\end{array}$ & $\begin{array}{l}\text { Jasminum polyanthum, Pink jasmine } \\
\text { Millettia reticulata, Evergreen wisteria } \\
\text { Trachelospermum jasminoides, Confederate jasmine } \\
\text { (See Flowering Vines for Florida (http://edis.ifas.ufl.edu/ } \\
\text { mg097) for additional vines) }\end{array}$ \\
\hline $\begin{array}{l}\text { Nandina } \\
\text { domestica } \\
\text { (species type or } \\
\text { wild type) }\end{array}$ & $\begin{array}{l}\text { Nandina, } \\
\text { Heavenly } \\
\text { bamboo }\end{array}$ & $\begin{array}{l}\text { Agarista populifolia, Florida leucothoe } \\
\text { Itea virginica, Virginia sweetspire }\end{array}$ & 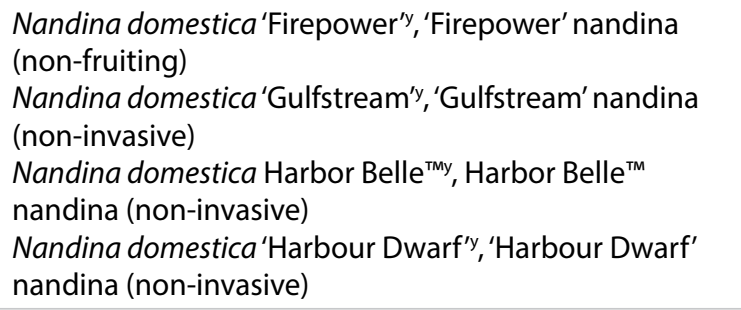 \\
\hline $\begin{array}{l}\text { Ruellia simplex ( } R \\
\text { brittoniana) }\end{array}$ & $\begin{array}{l}\text { Mexican } \\
\text { petunia }\end{array}$ & $\begin{array}{l}\text { Silphium asteriscus, Starry rosinweed } \\
\text { Sisyrinchium angustifolium, Blue-eyed grass } \\
\text { Stachytarpheta jamaicensis, Blue } \\
\text { porterweed } \\
\text { Stokesia laevis, Stokes' aster }\end{array}$ & $\begin{array}{l}\text { Ruellia simplex (formerly brittoniana) 'Purple Showers'y, } \\
\text { 'Purple Showers' Mexican petunia (sterile, non-invasive) } \\
\text { Eranthemum pulchellum, Blue sage } \\
\text { Plectranthus spp., Plectranthus } \\
\text { Plumbago auriculata, Plumbago } \\
\text { Ruellia simplex R10-102, Mayan Purple Mexican petunia } \\
\text { (sterile) } \\
\text { Ruellia simplex R10-108y, Mayan White Mexican petunia } \\
\text { (sterile) } \\
\text { Salvia farinacea, Mealycup sage } \\
\text { Salvia greggii, Autumn sage } \\
\text { Salvia leucantha, Mexican sage }\end{array}$ \\
\hline $\begin{array}{l}\text { Triadica sebifera } \\
\text { (syn. Sapium } \\
\text { sebiferum) }\end{array}$ & $\begin{array}{l}\text { Chinese tallow } \\
\text { tree, Popcorn } \\
\text { tree }\end{array}$ & $\begin{array}{l}\text { Acer rubrum, Red maple } \\
\text { Betula nigra, River birch }\end{array}$ & $\begin{array}{l}\text { Lagerstroemia spp., Crapemyrtle } \\
\text { Vitex agnus-castus, Chaste-tree }\end{array}$ \\
\hline $\begin{array}{l}\text { Schinus } \\
\text { terebinthifolius }\end{array}$ & Brazilian pepper & $\begin{array}{l}\text { Citharexylum spinosum, Fiddlewood } \\
\text { Hamelia patens, Firebush } \\
\text { Hydrangea quercifolia, Oakleaf hydrangea } \\
\text { Ilex cassine, Dahoon holly } \\
\text { llex vomitoria, Yaupon holly } \\
\text { Viburnum obovatum, Walter's viburnum }\end{array}$ & $\begin{array}{l}\text { Citrus spp., Citrus } \\
\text { Cocculus laurifolius, Laurel-leaf snailseed } \\
\text { llex cornuta, Chinese holly } \\
\text { Viburnum odoratissimum, Sweet viburnum } \\
\text { Viburnum odoratissimum var. awabuki, Awabuki } \\
\text { viburnum } \\
\text { Viburnum suspensum, Sandankwa viburnum }\end{array}$ \\
\hline $\begin{array}{l}\text { Sphagneticola } \\
\text { trilobata } \\
\text { (Wedelia } \\
\text { trilobata) }\end{array}$ & Wedelia & $\begin{array}{l}\text { Gaillardia pulchella, Firewheel } \\
\text { Helianthus debilis, Dune sunflower } \\
\text { Mimosa strigillosa, Powderpuff }\end{array}$ & $\begin{array}{l}\text { Arachis glabrata, Rhizoma perennial peanut } \\
\text { Euryops (formerly Gamolepis) chrysanthemoides, African } \\
\text { bush daisy } \\
\text { Ipomoea spp., Ornamental sweetpotato }\end{array}$ \\
\hline \multicolumn{4}{|c|}{$\begin{array}{l}\text { "As listed by the University of Florida/IFAS Status Assessment. The initial component of the IFAS Assessment of Non-Native Plants in Florida's } \\
\text { Natural Areas (IFAS Invasive Plant Working Group 2008) is the Status Assessment (Fox, Gordon, Dusky, Tyson, and Stocker 2009), in which } \\
\text { evidence is reviewed concerning ecological impacts, potential for expansion, difficulty of management, and economic value of non-native } \\
\text { species. } \\
\text { 'y } \\
\text { Gon-invasive cultivar derived from the invasive species as determined by the University of Florida/IFAS Infraspecific Taxon Protocol (Fox, } \\
\text { infraspecific taxa (e.g., cultivars, varieties, or subspecies) if these taxa can be clearly distinguished in the field and are not likely to revert. } \\
\text { Other infraspecific taxa (those indicated by this footnote) may be assessed using the Infraspecific Taxon Protocol (Fox, Gordon, Gantz, Knox, } \\
\text { and Wilson 2007). }\end{array}$} \\
\hline
\end{tabular}

